\title{
First look at time-dependent CP violation using early Belle II data
}

\author{
Daniel Červenkov*† \\ Charles University \\ E-mail: cervenkovaipnp.mff.cuni.cz
}

The Belle II experiment at the SuperKEKB energy-asymmetric $e^{+} e^{-}$collider is a substantial upgrade of the B factory facility at the Japanese KEK laboratory. In this paper, we report the first steps toward measurement of the time-dependent CP violation parameter for $B^{0}\left(\bar{B}^{0}\right) \rightarrow J / \psi K_{S}^{0}$ using an early Belle II data set. Furthermore, we briefly present a technique to improve $\alpha / \phi_{2}$ measurement and provide estimates of its uncertainty with the full Belle II data set.

XXIX International Symposium on Lepton Photon Interactions at High Energies - LeptonPhoton2019 August 5-10, 2019

Toronto, Canada

* Speaker.

${ }^{\dagger}$ On behalf of the Belle II Collaboration 


\section{Introduction}

$B$-factories supplied us with a range of remarkable results, including the first observation of $\mathrm{CP}$ violation outside of the kaon system and various measurements of the CKM parameters. Many of these measurements were made possible thanks to time-dependent analyses. What is more, these types of measurements are so important for their experimental programs that the accelerators and detectors themselves were specifically designed to enable time-dependent analyses. Figure 1 depicts the main ideas of time-dependent measurements at $B$-factories.

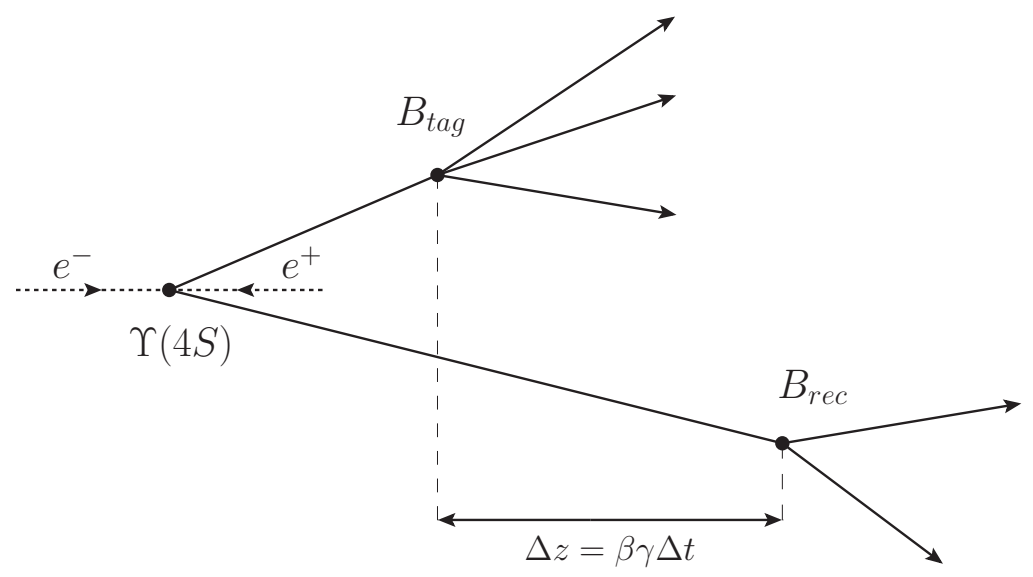

Figure 1: Principle of time-dependent measurement at $B$-factories

$B$-factories collide electrons and positrons, and their energies are tuned in such a way that $\Upsilon(4 S)$ resonances are often created. The $\Upsilon(4 S)$ is a bound state of $b$ and $\bar{b}$ quarks, and its mass, $10.58 \mathrm{GeV} / c^{2}$, is just above the $B \bar{B}$ meson production threshold of $10.56 \mathrm{GeV}$. Therefore, the majority of these resonances decay into a pair of $B$ mesons. When this happens, only about $20 \mathrm{MeV}$ is converted to kinetic energy of the daughter particles, i.e., the $B$ mesons are nearly at rest in the $\Upsilon(4 S)$ center-of-mass frame. $B$-factories take advantage of this fact, by boosting the $\Upsilon(4 S)$, which effectively means boosting both $B$ mesons in the same, well-known way. The actual technique that is used to achieve this boost is asymmetry in the beam energy between the positron and electron beams.

Because of the production mechanism, $B$ mesons at $B$-factories are created in anti-symmetrically entangled pairs. This essentially means that when the first $B$ meson decays via weak interaction as a flavor eigenstate, we know that in that instant, the other is in the opposite flavor eigenstate. This is paramount for time-dependent measurements, where the difference in decay times of the two mesons, $\Delta t=0$, is the main observable. $\Delta t$ as well as the flavors of the $B$ mesons at the time of their respective decays are the principal ingredients of time-dependent $\mathrm{CP}$ violation analyses at $B$-factories. Since the lifetime of $B$ mesons is relatively short, at the order of $1 \mathrm{ps}$, a very precise determination of $\Delta t$ is necessary. Figure 1 hints at how this is done. The detectors directly determine the decay vertex position of each $B$ meson, and their distance is used to calculate $\Delta t$. This is possible because the $B$ mesons are boosted, but nearly stationary relative to each other. Note that the angle between the two depicted $B$ meson paths is greatly exaggerated in Figure 1. Furthermore, 
the boost has another beneficial effect - time dilatation prolongs lifetimes of the particles in the lab frame, making the spatial separation of the vertices larger and, therefore, easier to measure.

\section{Vertex Position Measurement at Belle II}

Positions of the decay vertices at Belle II come mainly from two sub-detectors: the Pixel Detector (PXD) and the Silicon Vertex Detector (SVD). The PXD is the active element closest to the interaction point, at only $14 \mathrm{~mm}$ from the beams. It is a device based on DEPFET [1] technology and composed of small cells roughly $50 \times 60 \times 75 \mu \mathrm{m}$ in size. There is currently one layer installed in the Belle II detector. A second layer is planned to be added in 2021.

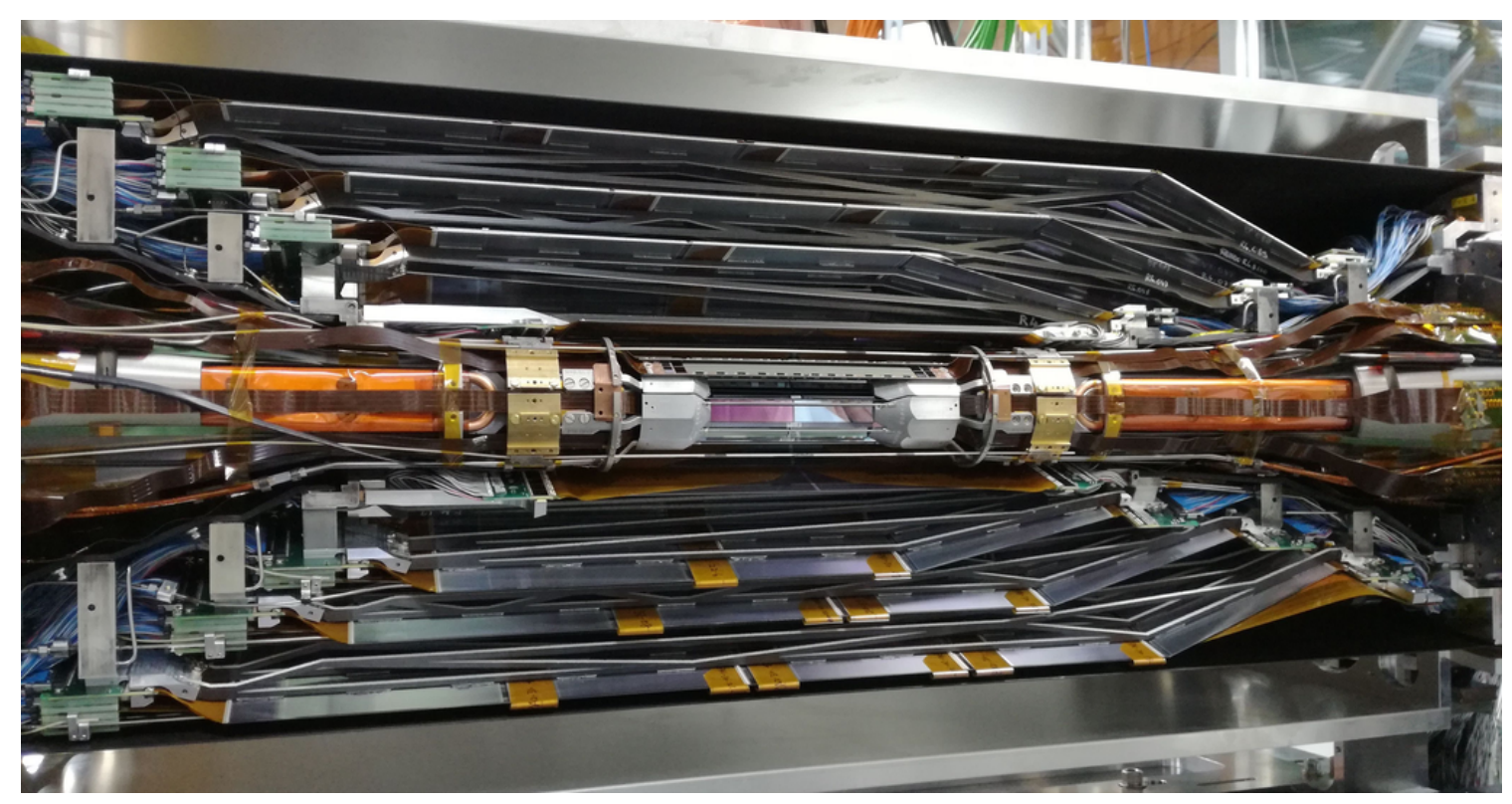

Figure 2: PXD (in the very center) and SVD in a mockup

The SVD is a silicon strip detector with four double-sided layers. It encircles the PXD, with the four layers that are distributed between 39 and $135 \mathrm{~mm}$ from the beams. It covers a sizable polar angle range - from $17^{\circ}$ to $150^{\circ}$. Elements of both sub-detectors, as well as their arrangement, can be seen in Figure 2.

It is worth mentioning that the SuperKEKB accelerator has a lower beam asymmetry and thus $B$ meson boost than its predecessor, KEKB. This is in order to decrease beam related backgrounds. The SuperKEKB $\beta \gamma=0.28$, which is about $2 / 3$ of the KEKB value. Since $\Delta t$ and the vertex spatial distance $\Delta z$ are related by $\Delta z=\beta \gamma \Delta t$, one would expect a lower $\Delta t$ resolution. However, the improved detectors more than compensate for this, as can be seen in Figure 3. Compare the $\sigma_{z}$ values with the average $|\Delta z| \approx 130 \mu \mathrm{m}[2]$.

\section{Measurement of $\beta / \phi_{1}$}

One of the mandates of $B$-factories is to measure the properties of electroweak CP violation. Constraining the angles of the standard unitarity triangle is one way of doing this. Currently, 


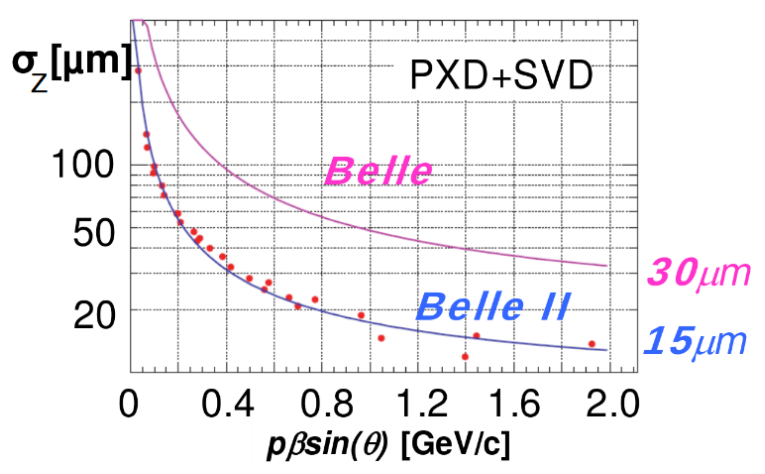

Figure 3: Comparison of $\Delta z$ resolution of Belle and Belle II

the most precise determination of angle $\beta / \phi_{1}$ comes from tree-level $b \rightarrow c$ processes, such as $B^{0} \rightarrow J / \psi K_{S}^{0}$. The full Belle II data sample will be so large, that uncertainty of these measurements will be dominated by systematics (mainly from alignment and $\Delta t$ resolution).

An independent determination of $\beta / \phi_{1}$ is possible using $b \rightarrow s q \bar{q}$ penguin-dominated processes, such as $B^{0} \rightarrow \eta^{\prime} K_{S}^{0}$. Penguin type processes incorporate loops, which effectively means they are suppressed relative to tree-level processes, but also that New Physics from a much higher energy scale can influence them. Therefore, it is prudent to compare results obtained from both types of decays. Such a comparison might look like Figure 4, where a simulated data set of a full Belle II data sample size was used. The parameter labeled $S$ quantifies the magnitude of time-dependent CP violation. Its projected uncertainty from Belle II measurements at various data sample sizes is shown in Table 1.

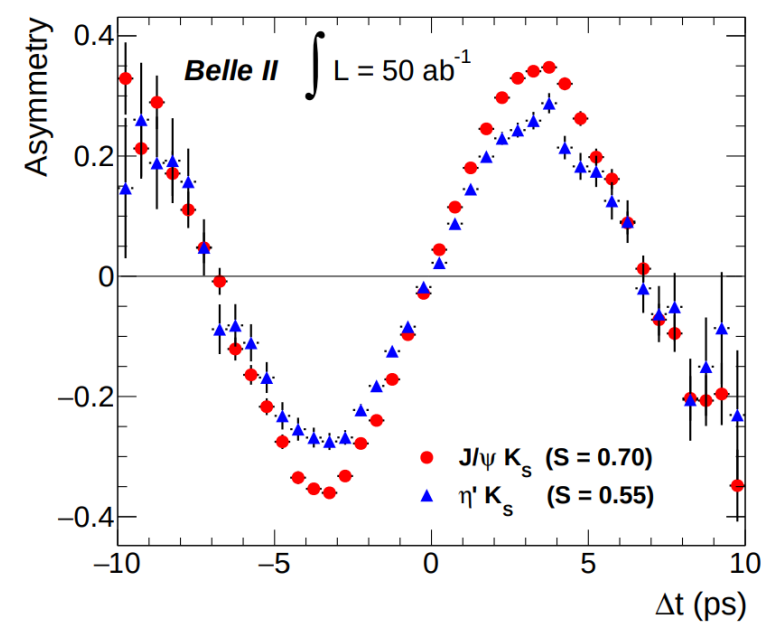

Figure 4: Simulated time-dependent asymmetry of two channels with a full Belle II data set

$B^{0} \rightarrow J / \psi K_{S}^{0}$ is the golden channel for time-dependent CPV. There are two reasons for this; firstly, a largish branching fraction $\left(8.7 \times 10^{-4}\right.$ for all $B^{0} \rightarrow J / \psi K^{0}$ channels combined), and secondly, negligible contributions from other than $b \rightarrow c \bar{c} s$ tree-level processes. The measurement of $\sin (2 \beta)$ is dominated by this channel. Furthermore, a very suitable control channel $B^{ \pm} \rightarrow J / \psi K^{ \pm}$ exists. It has an even larger branching fraction than the golden channel, and it has no CP violation, 


\begin{tabular}{llll}
\hline Channel & WA & $5 \mathrm{ab}^{-1}$ & $50 \mathrm{ab}^{-1}$ \\
\hline$J / \psi K_{S}^{0}$ & 0.022 & 0.012 & 0.0052 \\
$\eta^{\prime} K_{S}^{0}$ & 0.06 & 0.032 & 0.015 \\
$\Phi^{\prime} K_{S}^{0}$ & 0.12 & 0.048 & 0.020 \\
\hline
\end{tabular}

Table 1: Projection of Belle II uncertainty of $S$ at various luminosities (WA stands for current world average)

\begin{tabular}{lr}
\hline Type & \multicolumn{1}{c}{ Yield } \\
\hline$N_{\text {signal }}$ & $29.6 \pm 5.3$ \\
$N_{\text {background }}$ & $1.6 \pm 0.3$ \\
\hline
\end{tabular}

Table 2: Yield fit results of $B^{0} \rightarrow J / \psi K_{S}^{0}$ early Belle II sample

neither time-dependent nor direct, lending itself to checking analysis methods for any $\mathrm{CP}$ violation related bias.

Any $B$-factory analysis of time-dependent $\mathrm{CP}$ violation has to start with the selection and reconstruction of candidate particles. This was done using early Belle II data - about $2.62 \mathrm{fb}^{-1}$. A two-dimensional un-binned maximum likelihood fit was carried out to extract the number of signal and background events in the sample. The two variables of the fit in Figure 5 are

$$
\begin{aligned}
\Delta E & =E_{B}^{*}-E_{\text {beam }}^{*}, \\
M_{\mathrm{bc}} & =\sqrt{E_{\text {beam }}^{* 2}-\mathbf{p}_{B}^{* 2},}
\end{aligned}
$$

where $E_{B}^{*}$ and $\mathbf{p}_{B}^{*}$ stand for lab frame energy and momentum of the $B$ meson, respectively. $E_{\text {beam }}^{*}$ is the energy of the beam in the lab frame. Gaussian and ARGUS [3] functions were used for the probability density functions of the $M_{\mathrm{bc}}$ for the signal and background, respectively, while double Gaussian and linear functions were used for the $\Delta E$. The shaded regions were excluded from the fit to remove background candidates mainly due to $B^{0} \rightarrow J / \psi K^{* 0}$. The fit results can be seen in Table 2. One should keep in mind that flavor tagging is crucial for this analysis, and therefore the raw number of events does not tell the whole story. Compare the expected Belle II flavor tagging efficiency $\varepsilon_{\text {eff }}=36.67 \%$ [2] to a hadron machine experiment; LHCb flavor tagging efficiency is $\varepsilon_{\text {eff }}=3.02 \%[4]$.

\section{Measurement of $\alpha / \phi_{2}$}

The unitarity triangle angle $\alpha / \phi_{2}$ can be measured using isospin analysis of $B^{0} \rightarrow \pi \pi$ and $B^{0} \rightarrow \rho \rho$ families of decays. However, $B$-factories could not measure the time-dependent CP violation phase in $B^{0} \rightarrow \pi^{0} \pi^{0}$ decays, which caused an eightfold ambiguity in the angle. If one were to measure this phase, it would reduce the ambiguity by a factor of 2 or 4 , depending on the outcome. Measuring the phase essentially means doing a time-dependent analysis. As was mentioned before, $\Delta t$ is the principal observable in such analyses, and it is obtained by measuring positions of decay vertices. However, how does one measure the position of a $\pi^{0}$ decay? Belle II plans to use a two-pronged approach: firstly, it can simply use the Dalitz decays $\pi^{0} \rightarrow \gamma e^{+} e^{-}$, which 

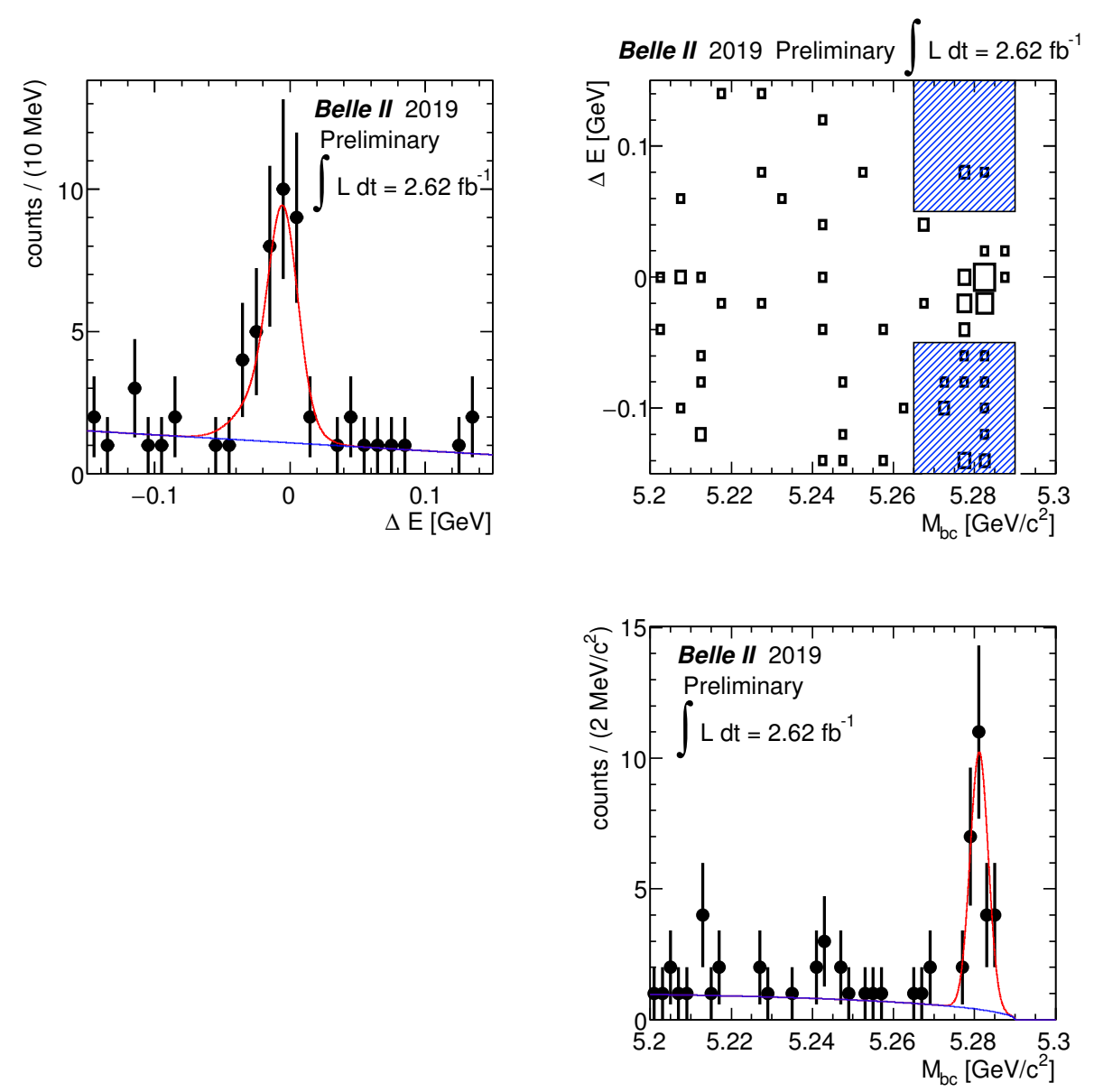

Figure 5: Two-dimensional unbinned ML yield fit of $B^{0} \rightarrow J / \psi K_{S}^{0}$ in early Belle II data

comprise about $1.2 \%$ of $\pi^{0}$ decays. Secondly, look for events where a $\gamma$ underwent $e^{+} e^{-}$conversion within the material of the beam pipe or PXD. Simulations show that the vertex resolution obtained from this method should be only about 50\% worse than that of a charged final state. That would still be an improvement over Belle charged final state resolution. With the full data set and all analyses of $B^{0} \rightarrow \pi \pi$ and $B^{0} \rightarrow \rho \rho$ decays, Belle II should determine $\alpha / \phi_{2}$ with a $0.6^{\circ}$ uncertainty.

\section{References}

[1] H.-G. Moser, The Belle II DEPFET pixel detector, Nuclear Instruments and Methods in Physics Research Section A: Accelerators, Spectrometers, Detectors and Associated Equipment 831 (2016) 85.

[2] E. Kou, P. Urquijo, W. Altmannshofer, F. Beaujean, G. Bell, M. Beneke et al., The Belle II Physics Book, 2018.

[3] H. Albrecht, R. Gläser, G. Harder, A. Krüger, A. Nilsson, A. Nippe et al., Search for hadronic $b \rightarrow u$ decays, Physics Letters B 241 (1990) 278.

[4] R. Aaij, B. Adeva, M. Adinolfi, A. Affolder, Z. Ajaltouni, S. Akar et al., Measurement of CP Violation in $B^{0} \rightarrow J / \psi K_{S}^{0}$ Decays, Physical Review Letters 115 (2015). 